\title{
A Fully Automated, Atlas-Based Approach for Superior Cerebellar Peduncle Evaluation in Progressive Supranuclear Palsy Phenotypes
}

\author{
(D) G. Nicoletti, (D).E. Caligiuri, (D) A. Cherubini, (D) M. Morelli, DF. Novellino, (D) G. Arabia, (D). Salsone, and (D) A. Quattrone
}

\begin{abstract}
BACKGROUND AND PURPOSE: The superior cerebellar peduncle is damaged in progressive supranuclear palsy. However, alterations differ between progressive supranuclear palsy with Richardson syndrome and progressive supranuclear palsy-parkinsonism. In this study, we propose an automated tool for superior cerebellar peduncle integrity assessment and test its performance in patients with progressive supranuclear palsy with Richardson syndrome, progressive supranuclear palsy-parkinsonism, Parkinson disease, and healthy controls.
\end{abstract}

MATERIALS AND METHODS: Structural and diffusion MRI was performed in 21 patients with progressive supranuclear palsy with Richardson syndrome, 9 with progressive supranuclear palsy-parkinsonism, 20 with Parkinson disease, and 30 healthy subjects. In a fully automated pipeline, the left and right superior cerebellar peduncles were first identified on MR imaging by using a tractography-based atlas of white matter tracts; subsequently, volume, mean diffusivity, and fractional anisotropy were extracted from superior cerebellar peduncles. These measures were compared across groups, and their discriminative power in differentiating patients was evaluated in a linear discriminant analysis.

RESULTS: Compared with those with Parkinson disease and controls, patients with progressive supranuclear palsy with Richardson syndrome showed alterations of all superior cerebellar peduncle metrics (decreased volume and fractional anisotropy, increased mean diffusivity). Patients with progressive supranuclear palsy-parkinsonism had smaller volumes than those with Parkinson disease and controls and lower fractional anisotropy than those with Parkinson disease. Patients with progressive supranuclear palsy with Richardson syndrome had significantly altered fractional anisotropy and mean diffusivity in the left superior cerebellar peduncle compared with those with progressive supranuclear palsy-parkinsonism. Discriminant analysis with the sole use of significant variables separated progressive supranuclear palsy-parkinsonism from progressive supranuclear palsy with Richardson syndrome with $70 \%$ accuracy and progressive supranuclear palsy-parkinsonism from Parkinson disease with 74\% accuracy.

CONCLUSIONS: We demonstrate the feasibility of an automated approach for extracting multimodal MR imaging metrics from the superior cerebellar peduncle in healthy subjects and patients with parkinsonian. We provide evidence that structural and diffusion measures of the superior cerebellar peduncle might be valuable for computer-aided diagnosis of progressive supranuclear palsy subtypes and for differentiating patients with progressive supranuclear palsy-parkinsonism from with those with Parkinson disease.

ABBREVIATIONS: $F A=$ fractional anisotropy; $M D=$ mean diffusivity; $P D=$ Parkinson disease; $P S P=$ progressive supranuclear palsy; PSP-P $=$ progressive supranuclear palsy-parkinsonism; PSP-RS = progressive supranuclear palsy with Richardson syndrome; SCP = superior cerebellar peduncle

$\mathbf{P}$ rogressive supranuclear palsy (PSP) is a neurodegenerative disorder characterized by a symmetric akinetic-rigid syndrome with vertical supranuclear palsy and falls. ${ }^{1}$ It is character-

Received January 18, 2016; accepted after revision October 24.

From the Institute of Bioimaging and Molecular Physiology (G.N., M.E.C., A.C., F.N., M.S., A.Q.), National Research Council, Catanzaro, Italy; and Institute of Neurology (M.M., G.A., A.Q.), University “Magna Graecia”, Catanzaro, Italy.

Please address correspondence to Giuseppe Nicoletti, MD, Institute of Bioimaging and Molecular Physiology, National Research Council, Viale Europa, 88100 Catanzaro, Italy; e-mail: g.nicoletti@unicz.it

Indicates article with supplemental on-line photo

http://dx.doi.org/10.3174/ajnr.A5048 ized by deposition of tau, with pathologic findings and degeneration affecting the white matter, particularly brain stem tracts, with less involvement of the cortex. ${ }^{2,3}$ Advanced neuroimaging studies using MR imaging ${ }^{4,5}$ and diffusion tensor imaging ${ }^{6,7}$ have confirmed the presence of a more severe involvement of white matter rather than cortical gray matter in PSP pathology. In particular, imaging alterations have been found in the superior cerebellar peduncles (SCPs), part of the dentatorubrothalamic tract that connects the dentate nucleus of the cerebellum to the ventrolateral thalamus, which in turn projects to the premotor cortex. Abnormal DTI measures of SCP were reported in patients with progressive supranuclear palsy with Richardson syndrome (PSP-RS). ${ }^{8}$ 
Other studies have found DTI alterations in the corpus callosum, internal capsules, and long-range white matter tracts. ${ }^{7,9-13}$ Recently, DTI metrics were used to distinguish the 2 variants of PSP,${ }^{14,15}$ the so-called progressive supranuclear palsy-parkinsonism (PSP-P), which is characterized by an asymmetric onset, resting tremor, poor response to levodopa, and PSP-RS, in which early falls and vertical supranuclear gaze palsy occur earlier than they do in the former variant. These studies agreed that MR imaging- and DTI-based metrics are able to reliably evaluate brain changes due to PSP in vivo, thus providing further insight into disease physiopathology. Because an error rate of $10 \%-30 \%$ has been reported in a clinical study with pathologic analysis, ${ }^{16}$ clinical criteria are not sufficient to make a correct diagnosis, especially at earlier stages of illness. The use of these quantitative measures in clinical practice would help improve the accuracy of the diagnostic process, especially in the attempt to have early differentiation of parkinsonian syndromes or the 2 disease phenotypes. However, the extraction of these metrics is not straightforward in everyday clinical practice because of the advanced techniques that need to be implemented; hence, an easy method to quickly obtain relevant quantities would be very useful in the diagnostic work-up.

The authors aimed to use a simple, atlas-based tool for the automatic assessment of SCP volume and microstructural integrity; this approach is very useful to correctly diagnose patients with parkinsonian syndromes.

\section{MATERIALS AND METHODS Patients}

This study was approved by the local ethics committee of our institution (Institute of Neurology, University Magna Graecia of Catanzaro, Italy), and all subjects provided written informed consent before enrollment. Thirty subjects who met the clinical research criteria for probable or possible $\mathrm{PSP}^{17}$ and 20 with a diagnosis of Parkinson disease $(\mathrm{PD})^{18}$ (mean age, $66.2 \pm 3.0$ years) were included in this study. The PSP group was divided into 9 patients with PSP-P (mean age, $70.1 \pm 4.8$ years) and 21 with PSP-RS (mean age, $71.9 \pm 5.9$ years). All patients were examined at the Institute of Neurology, University Magna Graecia, Catanzaro, Italy, between June 2013 and June 2014, by a movement disorders specialist and PSP expert (G.N.). All subjects underwent detailed clinical evaluations. The patient's disability was assessed by using the Unified Parkinson's Disease Rating Scale-Motor Examination, ${ }^{19}$ and disease severity, by using the Hohen and Yahr scale. ${ }^{20}$ Onset of falls and supranuclear gaze palsy within 2 years was associated with a diagnosis of PSP-RS, while patients were diagnosed as having PSP-P if they presented with oculomotor slowness or backward falls at 2 years from disease onset, asymmetry of limb signs, and moderate/good improvement in bradykinesia and rigidity after levodopa administration. All patients with PSP-RS had probable PSP; 7 patients with PSP-P had probable PSP, and 2 patients with PSP-P had possible PSP. When MR imaging was performed, all patients with PSP-P were classified as PSP-P, even though the onset of the disease in some patients could be confused with an idiopathic PD. To examine cognitive functions, we administered the Mini-Mental State Examination. ${ }^{21}$ Thirty healthy subjects were also recruited. All controls per- formed within normal limits on standardized neurologic and neuropsychological testing.

\section{MR Imaging Acquisition and Processing}

All participants underwent the same MR imaging protocol. Patients were examined by using a 3T Discovery MR750 scanner (GE Healthcare, Milwaukee, Wisconsin). The MR imaging protocol included whole-brain, 3D, T1-weighted (BRAVO; GE Healthcare, Milwaukee, Wisconsin), spoiled gradient recalled-echo imaging (TE/TR $=3.7 /$ $9.2 \mathrm{~ms}$, flip angle $=12^{\circ}$, voxel size $=1 \times 1 \times 1 \mathrm{~mm}^{3}$ ), diffusion tensor imaging $\left(b=1000 \mathrm{~s} / \mathrm{mm}^{2}\right.$, diffusion-weighting along 27 noncollinear gradient directions, matrix size $=128 \times 128$, 80 axial sections, number of $b=0$ images $=4$, NEX $=2$, voxel size $=2 \times 2 \times 2$ $\mathrm{mm}^{3}$ ), and fast fluid-attenuated inversion recovery axial images (TR/ $\mathrm{TE}=9500 / 100 \mathrm{~ms}$, matrix size $=512 \times 512, \mathrm{FOV}=24 \mathrm{~cm}$, thirtysix 4-mm sections, gap $=0 \mathrm{~mm}$ ).

FLAIR images were visually checked to assess vascular lesions in each patient. The extent and possible etiology of white matter hyperintensities were not different across patients, independent of the group. Moreover, none of the participants showed infratentorial lesions that could affect volumetric and diffusion measures in the SCP.

A graphic description of our fully automated processing workflow is shown in Fig 1. Image processing was performed by using FSL (http://www.fmrib.ox.ac.uk/fsl). ${ }^{22}$ Brain tissue volume, normalized for subject head size, was estimated with the SIENAX tool (http://fsl.fmrib.ox.ac.uk/fsl/fslwiki/SIENA). ${ }^{23,24}$ SIENAX starts by extracting brain and skull images from the single whole-head input data. ${ }^{25}$ The brain image is then affine-registered to Montreal Neurological Institute-152 space (by using the skull image to determine the registration scaling); this processing step is primarily to obtain the volumetric scaling factor, to normalize subsequently extracted measures for head size. Moreover, SIENAX provides the partial volume estimates for the different tissues in the brain. In particular, in this study, we exploited the white matter partial volume estimates to exclude CSF voxels from the analysis, as will be further explained in the next section.

Head motion and image distortions induced by eddy currents in the DTI data were corrected by applying a 3D full-affine (mutual-information cost function) alignment of each image to the mean no-diffusion-weighting (B0) image. After distortion correction, DTI data were averaged and concatenated into 28 (1 mean B0 + 27 B1000) volumes. A diffusion tensor model was fit at each voxel, generating fractional anisotropy (FA) and mean diffusivity (MD) maps. The FA maps were then registered to wholebrain-extracted T1-weighted images by using a full-affine (correlation-ratio cost function) alignment with nearest neighbor resampling. The calculated transformation matrix was then applied to the MD maps with identical resampling options. ${ }^{26}$

\section{ROI Extraction}

To localize ROIs for the left and right SCPs on the T1 images and coregistered DTI maps of each subject, we used the tractographybased atlas of human brain connections (http://www.natbrainlab. com/), obtained from tractography data of 40 healthy adults mapped onto a common reference space (Montreal Neurological Institute). ${ }^{27}$ This atlas provides probability maps of each recon- 


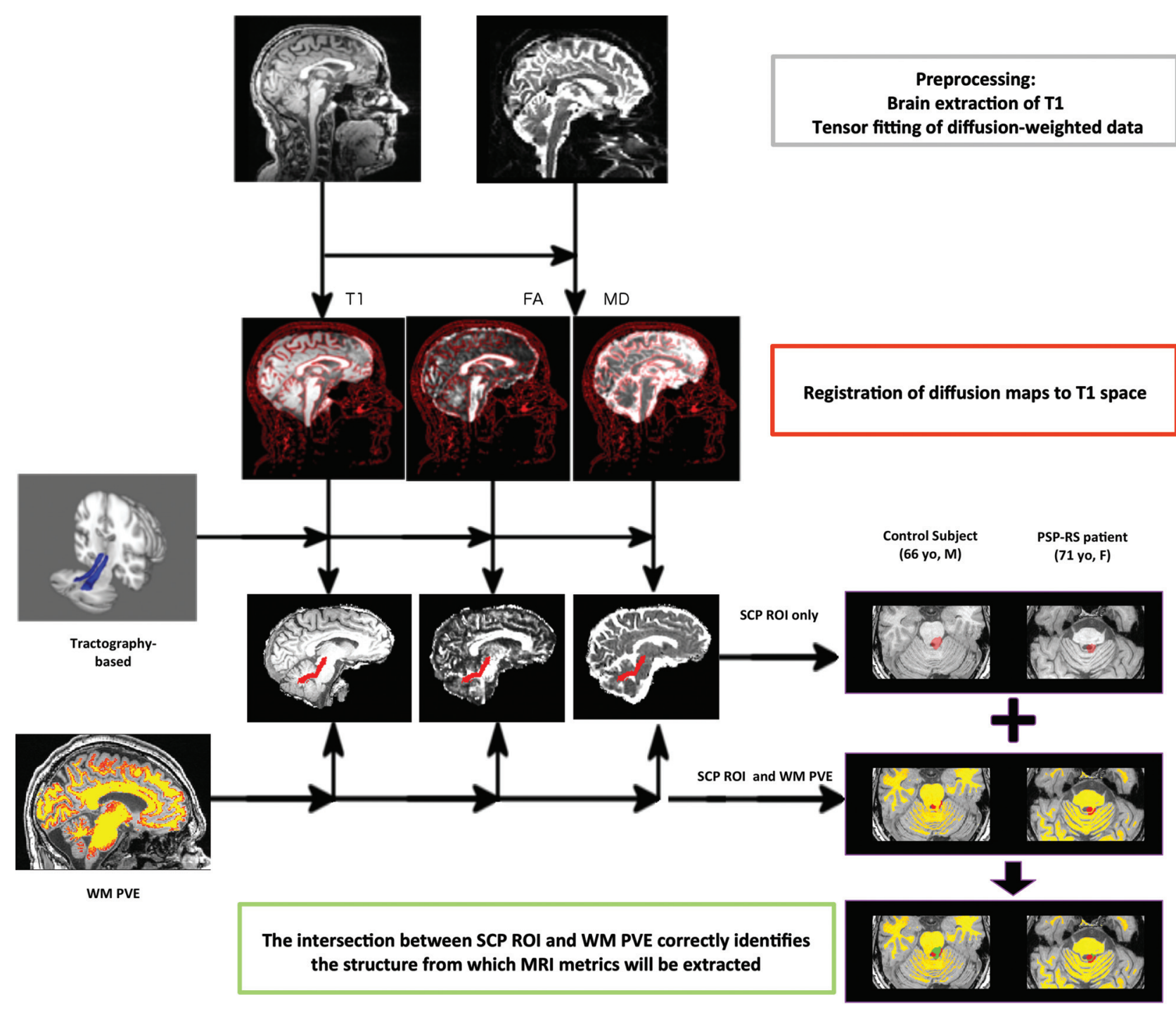

FIG 1. Image-processing workflow. The coregistration of different sequences allows the extraction of multimodal parameters from an ROI.

structed bundle: each voxel value ranges from 0 to 1 and represents the proportion of subjects in which that same voxel was part of the bundle. Thus, we thresholded the bilateral SCP probability maps at 0.3 so that each included voxel was represented in at least $30 \%$ of the subjects from whom the atlas was obtained. This threshold was chosen on the basis of the overlap of the ROI with the anatomic regions in the template.

Subsequently, ROIs were warped in each subject's T1 space in the following manner: First, the Montreal Neurological Institute152 template was nonlinearly registered to each subject's T1 image by using the FMRIB Nonlinear Registration Tool (FNIRT; http://fsl.fmrib.ox.ac.uk/fsl/fslwiki/FNIRT); afterward, the resulting warp field was applied to the SCP binary masks. Before extracting MR imaging metrics from selected ROIs, we performed a processing step to account for confounding effects due to CSF contamination. In particular, each subject's WM partial volume estimate was thresholded at 0.75 , to retain only those voxels that belonged to WM with a probability of at least $75 \%$. The resulting mask was then used, in combination with the SCP ROIs, to automatically extract right and left SCP volumes, average FAs, and average MDs for each subject.
A 2-step quality check was performed to ensure validity of the image-processing pipeline: First, the contrast-to-noise ratio of the included scans had to be excellent; second, to exclude CSF contamination, an expert visually inspected the outcome of nonlinear registration between ROIs and T1-weighted images.

\section{Statistical Analyses}

The difference in sex distribution between patients and control subjects and among groups of patients with different movement disorders was evaluated with the $\chi^{2}$ test. The Shapiro-Wilk test was used to assess normal distribution of continuous variables. Differences in normal clinical variables among the study groups were assessed by using 2-tailed, 2-sample $t$ tests, while the MannWhitney $U$ test was used for non-normally distributed variables. The threshold for statistical significance was set at .05 after Bonferroni correction for multiple comparisons. Differences in the multimodal imaging variables across groups were evaluated by analysis of variance, with age, sex, and disease duration as covariates. The Tukey honest significant difference test was used to identify pair-wise differences between groups, corrected for multiple comparisons. Pearson correlation analysis was used to eval- 
Table 1: Demographic, clinical, and neuroimaging features in patients with PSP-P, PSP-RS, PD, and healthy controls

\begin{tabular}{lcccc}
\hline & PSP-P $(\boldsymbol{n}=\mathbf{9})$ & PSP-RS $(\boldsymbol{n}=\mathbf{2 1})$ & PD $(\boldsymbol{n}=\mathbf{2 0})$ & HC $(\boldsymbol{n}=\mathbf{3 0})$ \\
\hline Age (mean) (yr) & $70.1 \pm 4.8$ & $71.9 \pm 5.9$ & $66.2 \pm 3.0$ & $67.2 \pm 7.2$ \\
Sex, \% male & $100 \%$ & $57 \%$ & $50 \%$ & $47 \%$ \\
Disease duration (mean) (yr) & $6.3 \pm 3.7$ & $3.1 \pm 1.4$ & $7.5 \pm 3.7$ & - \\
MMSE (mean) & $22.1 \pm 3.6$ & $19.0 \pm 5.4$ & $23.2 \pm 3.3$ & - \\
UPDRS-III (mean) & $22.8 \pm 17.7$ & $39.3 \pm 7.7$ & $27.1 \pm 9.1$ & - \\
HY (mean) & $2.9 \pm 0.7$ & $3.4 \pm 0.8$ & $2.2 \pm 0.5$ & - \\
SCP right & & & \\
$\quad$ Volume (No. of voxels) (mean) & $5652 \pm 1172$ & $5282 \pm 753$ & $6907 \pm 1306$ & $6569 \pm 944$ \\
FA (mean) & $0.44 \pm 0.05$ & $0.40 \pm 0.05$ & $0.48 \pm 0.04$ & $0.45 \pm 0.02$ \\
MD (X10 mm $^{2} / \mathrm{s}$ ) (mean) & $0.873 \pm 0.06$ & $0.949 \pm 0.170$ & $0.851 \pm 0.089$ & $0.833 \pm 0.031$ \\
SCP left & & & & \\
Volume (No. of voxels) (mean) & $5315 \pm 1121$ & $4812 \pm 701$ & $6369 \pm 1205$ & $5961 \pm 894$ \\
FA (mean) & $0.44 \pm 0.04$ & $0.39 \pm 0.05$ & $0.47 \pm 0.04$ & $0.45 \pm 0.02$ \\
MD ( $\times 10^{-3} \mathrm{~mm}^{2} / \mathrm{s}$ (mean) & $0.895 \pm 0.061$ & $0.974 \pm 0.074$ & $0.874 \pm 0.066$ & $0.857 \pm 0.023$ \\
\hline
\end{tabular}

Note:-HC indicates healthy controls; MMSE, Mini-Mental State Examination; UPDRS-III, Unified Parkinson's Disease Rating Scale-Motor Examination; HY, Hohen and Yahr score.

uate the relationship between MR imaging parameters and clinical variables. All statistical analyses were performed with $\mathrm{R}$ software (http://www.R-project.org).

\section{Linear Discriminant Analysis}

After performing comparisons of the MR imaging variables across different groups, we studied the discriminant power of these measures. In particular, we applied linear discriminant analysis on our dataset, to identify the following: which variables perform better in separating the groups and what is the predictive power of these measures (ie, how is a new subject classified on the basis of these measures?). We can define a total covariance matrix $\mathrm{C}$, as the combination of 2 components:

1) The between-subjects covariance matrix (B), which represents the covariance of the different variable means.

2) The within-subjects covariance matrix (W), which represents the covariance of the distances between individual values and group means.

This relationship is expressed by the following equation: $\mathrm{C}=\mathrm{B}+\mathrm{W}$, which is a generalization of 1-way analysis of variance in the case of a dataset with multiple variables. In this context, a discriminant analysis searches for a combination of variables that maximize either the $\mathrm{B} \times \mathrm{C}^{-1}$, in which case the approach is descriptive and the constraint is that the total variance $(C)$ of the linear combination of variables equals 1 , or the $\mathrm{B} \times \mathrm{W}^{-1}$ term, in which case the approach is predictive and the within variance $(\mathrm{W})$ of the correlation equals 1 .

In this study, we first performed the analysis in descriptive mode on a dataset comprising all MR imaging metrics, measured on all the study participants, divided according to the diagnosis. Subsequently, we tested the predictive approach by building 2 different models that included only the variables that were significant in group-wise comparisons. In particular, we were interested in identifying which variables could better differentiate the 2 PSP phenotypes (first model) or PSP-P and PD (second model). In both cases, leave-one-out cross-validation was used. Leaveone-out cross-validation works as follows: At each iteration, the linear discriminant model is trained on all subjects except 1 , which is used to test the predictive power of the model. The accuracy is computed across all iterations and is used to evaluate the model.

\section{RESULTS}

\section{Patients}

Table 1 shows the demographic and clinical characteristics of the patients. At the examination, age was higher in the patients with PSP-RS compared with healthy controls $(P=.02)$ and patients with PD $(P=.01)$. Those with PSP-RS also had significantly shorter disease duration compared with those with $\mathrm{PD}(P=$ $.0001)$ and PSP-P $(P=.02)$. Differences in Hohen and Yahr stages were found in those with PD $(P=.0004)$ and PSP-RS $(P=.001)$ compared with patients with PSP-P. Subjects with PSP-RS had significantly higher Unified Parkinson's Disease Rating ScaleMotor Examination scores compared with those with PSP-P $(P=$ $.002)$ and $\mathrm{PD}(P=.0004)$.

There was no significant correlation between clinical and imaging variables. The only significant correlation surviving correction for multiple comparisons was the one found in patients with PD between the Hohen and Yahr score and age $(r=0.78, P<.05)$.

\section{ROI Analysis}

Table 1 also summarizes values of volume, FA, and MD of the right and left SCPs in the different groups. Figure 2 shows the boxplots for the MR imaging metrics that were considered in the analysis. $P$ values for the different comparisons can be found in Table 2.

Both PSP subtypes showed significant damage to the SCP. In particular, patients with PSP-RS showed alterations of all metrics (decreased volume, decreased FA, and increased MD) bilaterally compared with patients with PD and control subjects. Patients with PSP-P had a bilateral SCP volume decrease compared with controls and decreased SCP volume and FA bilaterally compared with those with PD.

In the comparison between PSP subtypes, we found significant differences in the left SCP. In particular, patients with PSP-RS had significantly decreased FA values $(P=.007)$ and significantly increased MD values $(P=.003)$ in this structure.

Finally, we found an increase in FA in patients with PD compared with controls, in the right SCP.

\section{Linear Discriminant Analysis}

On-line Fig 1 shows a graphic representation of the descriptive discriminant analysis. In particular, each subject is projected on a 
Left SCP Volume

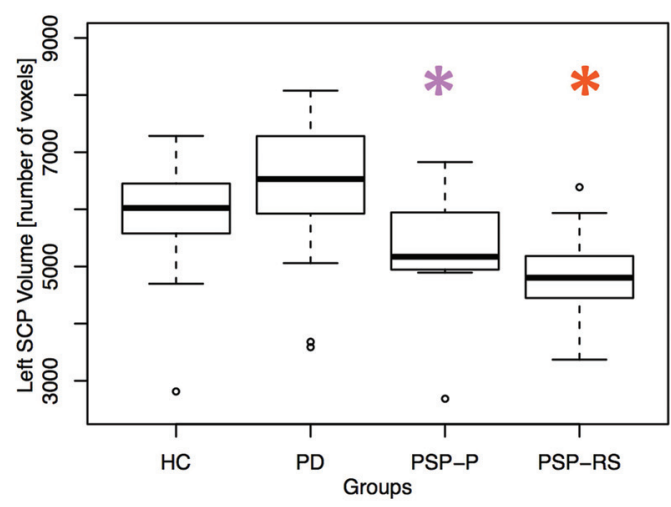

Left SCP Fractional Anisotropy

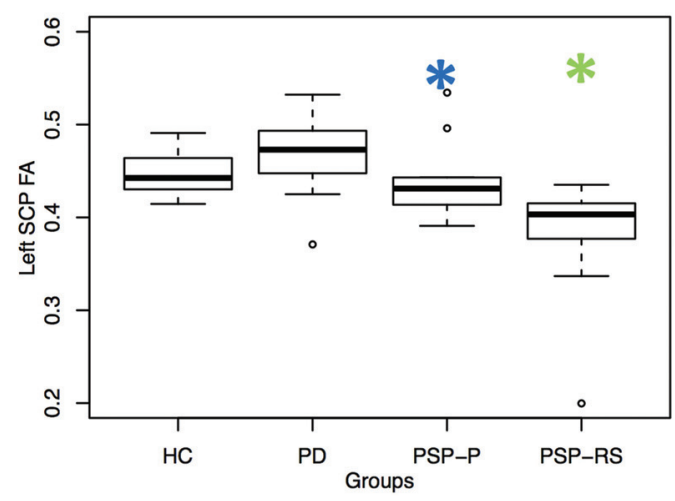

Left SCP Mean Diffusivity

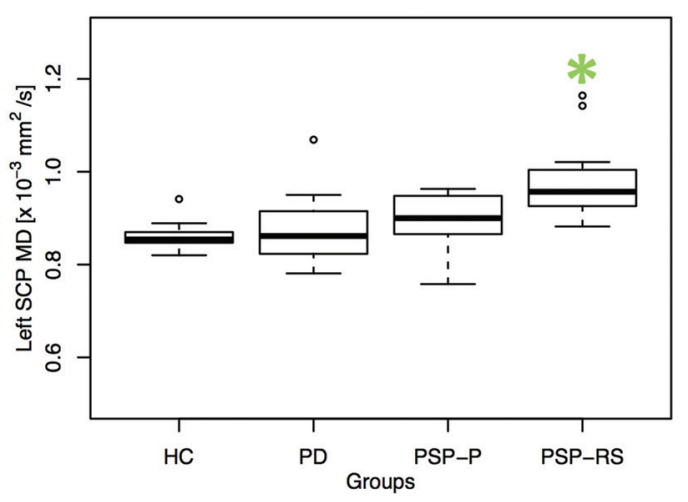

Right SCP Volume

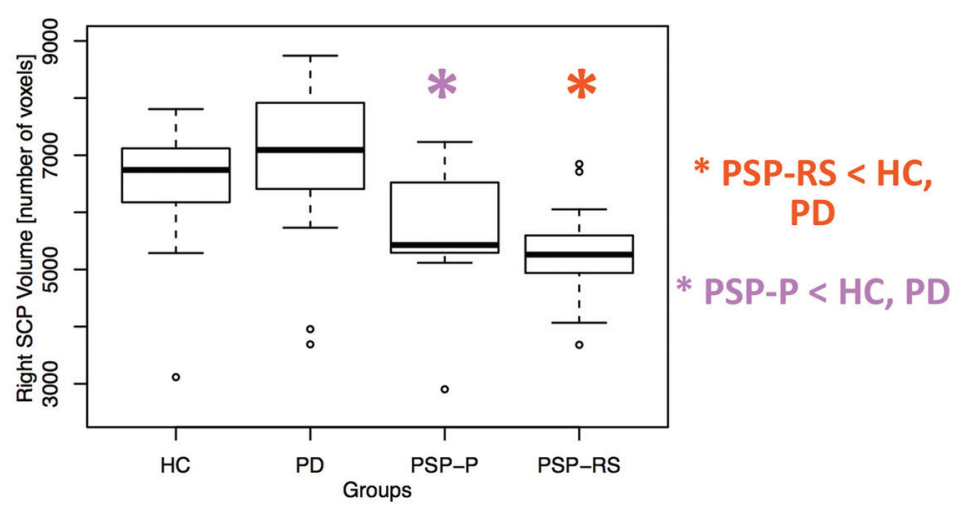

Right SCP Fractional Anisotropy

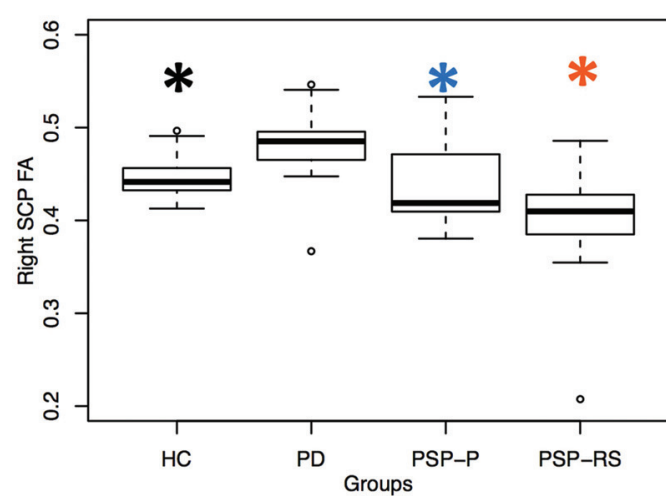

* PSP-RS < HC, PD, PSP-P

* PSP-P < PD

* PSP-RS < HC, PD

$* \mathrm{PD}>\mathrm{HC}$
Right SCP Mean Diffusivity

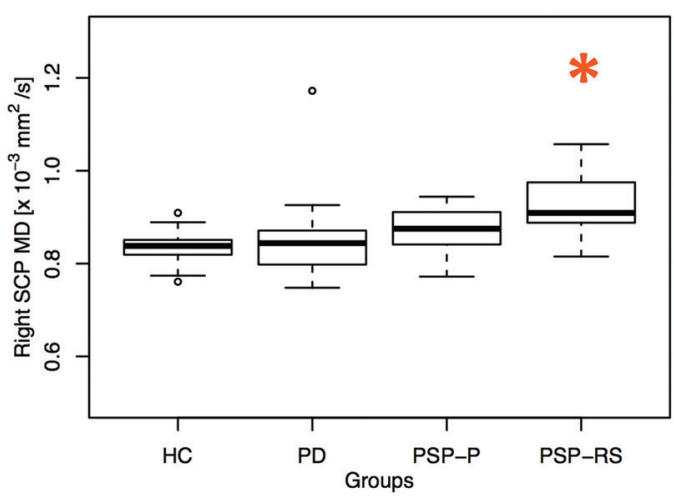

* PSP-RS > HC, PD, PSP-P

* PSP-RS > HC, PD

FIG 2. Box-and-whisker plots of volumes, FA, and MD of the right and left SCPs in patients and controls.

plane defined by the linear discriminant components. Each group is represented by an ellipse. The ellipse center indicates the means (between-variances), while the ellipse area is proportional to within-variances.

In the first predictive discriminant analysis model, we included FA and MD values from the left SCP because they were significantly different between the 2 PSP phenotypes. The accuracy of the model reached $70 \%$, with $\mathrm{MD}$ as the best predictor (coefficients of the linear discriminant for $\mathrm{MD}=9.67$ and for $\mathrm{FA}=-7.87)$. In the second model, instead, volume and FA from bilateral SCPs were used to discriminate patients with PD from those with PSP-P. This model reached an accuracy of 74\%, with FA values performing better than volume in separating the 2 groups (coefficients of the linear discriminant analyses: -29.6 and 18.7 for FA of right and left SCPs, respectively; -0.002 and 0.001 for right and left SCP volumes, respectively).

\section{DISCUSSION}

In the present study, we introduced a fully automated pipeline for the assessment of SCP integrity in patients with PSP, PD, and healthy subjects. The proposed method takes advantage of an atlas-based ROI approach that allowed the automated individuation of the SCP, thus avoiding time-consuming and highly userdependent manual measurements. We also tested the ability of this tool to distinguish the 2 PSP subtypes. By assessing volume and diffusion metrics of the SCP, we found significant differences 
Table 2: $P$ values from the statistical tests among different groups after correction for multiple comparisons (Tukey test) ${ }^{a}$

\begin{tabular}{|c|c|c|c|c|c|c|}
\hline & PD vs HC & PSP-P vs HC & PSP-RS vs HC & PSP-P vs PD & PSP-RS vs PD & PSP-P vs PSP-RS \\
\hline Age & 1 & 1 & $.02^{\mathrm{b}}$ & 0.1 & $.01^{\mathrm{b}}$ & 1 \\
\hline Sex & .1 & $.0001^{\mathrm{b}}$ & .1 & $.008^{b}$ & 1 & $.01^{\mathrm{b}}$ \\
\hline Disease duration & - & - & - & 1 & $.0001^{b}$ & $.02^{b}$ \\
\hline MMSE & - & - & - & 1 & $.01^{\mathrm{b}}$ & 1 \\
\hline UPDRS-III & - & - & - & 1 & $.0022^{\mathrm{b}}$ & $.002^{b}$ \\
\hline HY & - & - & - & $.0004^{\mathrm{b}}$ & .94 & $.001^{\mathrm{b}}$ \\
\hline \multicolumn{7}{|l|}{ SCP right } \\
\hline Volume & .66 & $.008^{\mathrm{b}}$ & $.00005^{b}$ & $.001^{\mathrm{b}}$ & $.000004^{b}$ & .95 \\
\hline FA & $.01^{\mathrm{b}}$ & .66 & $.0005^{b}$ & $.004^{b}$ & $1.45 \times 10^{-8 b}$ & .16 \\
\hline$M D$ & .89 & .38 & $.0003^{b}$ & .77 & $.01^{\mathrm{b}}$ & .31 \\
\hline \multicolumn{7}{|l|}{ SCP left } \\
\hline Volume & .44 & $.03^{b}$ & $.00008^{b}$ & $.002^{b}$ & $.000002^{b}$ & .81 \\
\hline FA & .15 & .58 & $.0000005^{b}$ & $.03^{\mathrm{b}}$ & $9.33 \times 10^{-10 b}$ & $.007^{\mathrm{b}}$ \\
\hline$M D$ & .70 & .16 & $9.30 \times 10^{-10 b}$ & .67 & $.000001^{\mathrm{b}}$ & $.003^{b}$ \\
\hline
\end{tabular}

Note:- HC indicates healthy controls; MMSE, Mini-Mental State Examination; UPDRS-III, Unified Parkinson's Disease Rating Scale-Motor Examination; HY, Hohen and Yahr score.

a Sex differences were assessed with $\chi^{2}$ tests. Age differences were assessed with ANOVA. Disease duration differences were assessed using ANCOVA, with age and sex as covariates. Other clinical and imaging differences were assessed with ANCOVA, with age, sex, and disease duration, as covariates. Correction for multiple comparisons was performed with the Tukey honest significant difference test.

bignificant.

between PSP-P and PSP-RS in MD and FA of the left SCP, with the latter phenotype more severely damaged. As expected, both PSP phenotypes showed extensive alterations of multimodal MR imaging metrics compared with patients with PD and controls. Discriminant analysis of the significantly different metrics allowed separation of the 2 PSP subtypes with an accuracy of $70 \%$ and could also distinguish PSP-P from PD with an accuracy of $74 \%$.

By using a quick and fully automated pipeline, we were able to analyze a predefined ROI (ie, SCP) on different MR imaging sequences, thus collecting information in different scales (ie, macroscopic volume and microstructural integrity). SCP identification and evaluation were performed by an atlas-based approach. The use of an atlas has multiple benefits. First, it facilitates the identification of brain structures on MR imaging in healthy subjects and patients. ${ }^{27}$ Second, it avoids a manual ROI definition, which is a time-consuming and strongly user-dependent procedure; this feature raised the level of reproducibility of this study. Despite heavy SCP damage present in PSP, which could hamper the identification of the structure, the quality check of images performed in this study confirmed that the grade of superimposition between the SCP masks and the T1 images was appropriate.

The choice of the ROI for analyses was because atrophy of the SCP is a well-known postmortem finding in patients with PSP. ${ }^{3}$ This bundle is composed of efferent cerebellar fibers, mainly originating from the dentate nucleus, which decussate and project via the red nucleus to the contralateral ventrolateral nucleus of the thalamus. SCP fibers that project to the reticular and vestibular nuclei of the brain stem may be involved in the pathophysiology of postural instability in PSP. Moreover, damage of SCP fibers that contributes to the control of smooth pursuit movements may contribute to gaze palsy in this disorder. ${ }^{28}$ In previous studies, we investigated MR imaging alterations in SCP and infratentorial structures of patients with PD, PSP, and multiple system atrophy with predominant parkinsonian signs, ${ }^{29-31}$ but we had not yet considered the 2 PSP phenotypes separately.

Two recent studies have found differences between PSP-RS and PSP-P by using volumetry of brain stem structures ${ }^{14}$ or DTI metrics. ${ }^{15}$ In the former, the authors concluded that SCP was relatively spared in patients with PSP-P, but not in those with PSP-RS; in the latter, instead, the authors demonstrated the utility of combining DTI metrics to the well-known Magnetic Resonance Parkinsonism Index. ${ }^{31}$ Our findings are in line with results from both studies, because patients with PSP-RS were found more severely damaged in most comparisons and DTI metrics helped uncover differences between subtypes that could not be found by volume alone.

In this study, patients with PSP-RS showed bilateral alterations of all SCP metrics compared with controls and patients with $\mathrm{PD}$, whereas compared with patients with PSP-P, they showed altered diffusion metrics in the left SCP only. This finding suggests that the right SCP might be equally damaged between the 2 phenotypes, while the left SCP seems to be relatively spared in PSP-P. The presence of unilateral significant differences in SCP between PSP-P and PSP-RS is not surprising and is in line with the asymmetric clinical presentation of PSP-P. ${ }^{32}$ The presence of left-sided damage in PSP-RS is also in line with results from a recent study investigating white matter loss in PSP. ${ }^{33}$

The integrity of the SCP, which characterized patients with PD in this study, supported the robustness of our automated method, confirming the notion that this structure is not involved in PD. Increased MD and decreased FA values in the SCP in PSP compared with $\mathrm{PD}$ have also been reported previously. ${ }^{34,35}$ Patients with PD showed increased FA in the SCP compared not only with patients with PSP but also with healthy controls. Despite pathologic alterations being usually associated with decreases of this metric, increases of FA have also been reported and are thought to characterize a selective degeneration of white matter bundles in regions of crossing fibers. ${ }^{36}$ Thus, in the present study, the higher FA found in patients with PD could be the result of different pathologic processes that do not hamper the microstructural integrity of the entire SCP as we found in PSP-RS but rather cause the loss of connections with an orientation different from the principal diffusion direction of the SCP.

Results of the discriminant analysis between PSP phenotypes seem to encourage the adoption of this automated approach in 
clinical practice, though at the present time, this is not yet feasible. In fact, validation is still needed on larger cohorts and, at the same time, in assessing the reliability of the method in individual subjects, possibly with confirmed postmortem diagnosis. Despite these well-known limitations, however, the accuracy of $70 \%$ obtainable with the sole use of diffusion metrics extracted from the left SCP suggests that our tool could be valuable in the clinical diagnostic process, possibly integrated with other measures that currently aid the diagnosis (eg, the Magnetic Resonance Parkinsonian Index).

Overall, our findings demonstrate the following: 1) Automatic extraction of multimodal MR imaging metrics from the SCP is feasible not only in healthy controls but also in patients with PD and PSP; 2) damage to the SCP is present in the 2 forms of PSP with a different degree of severity: more severe in PSP-RS than in PSP-P, despite the significantly longer disease duration in the latter form; 3) SCP metrics in patients with PD were comparable with those extracted from healthy subjects; 4) the in vivo microstructural changes observed in SCP with DTI are in line with abnormalities detected in previous postmortem studies; 5) the degenerative process seems to begin on one side of the SCP and then progresses in the contralateral structure; and 6) damage to the SCP as detected by volume and FA might improve differentiation of PSP-P and PD in an early stage of the disease.

There are some limitations to our study: First, the population was relatively small. Second, we did not have postmortem confirmation to reach the criterion standard diagnosis and cannot fully exclude misdiagnosis. Third, the tool still needs validation on larger cohorts of patients and on MR imaging scans acquired with different parameters.

\section{CONCLUSIONS}

With a fully automated analysis pipeline, we were able to rapidly extract MR imaging markers that helped identify different patterns of SCP damage, not only between PSP-P and PSP-RS but also between PSP-P and PD. This approach was implemented with minimal user intervention, which guarantees reproducibility of the results and avoids the time-consuming procedures required for manual segmentation of ROIs. The proposed pipeline could be useful if integrated into the current diagnostic process, to improve early diagnosis of PD and different parkinsonian syndromes, especially in the most ambiguous cases.

\section{REFERENCES}

1. Steele JC, Richardson JC, Olszewski J. Progressive supranuclear palsy: a heterogeneous degeneration involving the brain stem, basal ganglia and cerebellum with vertical gaze and pseudobulbar palsy, nuchal dystonia and dementia. Arch Neurol 1964;10:333-59 CrossRef Medline

2. Hauw JJ, Daniel SE, Dickson D, et al. Preliminary NINDS neuropathologic criteria for Steele-Richardson-Olszewski syndrome (progressive supranuclear palsy). Neurology 1994;44:2015-19 CrossRef Medline

3. Tsuboi Y, Slowinski J, Josephs KA, et al. Atrophy of superior cerebellar peduncle in progressive supranuclear palsy. Neurology 2003;60: 1766-69 CrossRef Medline

4. Josephs KA, Whitwell JL, Dickson DW, et al. Voxel-based morphometry in autopsy proven PSP and CBD. Neurobiol Aging 2008;29: 280-89 CrossRef Medline
5. Price S, Paviour D, Scahill R, et al. Voxel-based morphometry detects patterns of atrophy that help differentiate progressive supranuclear palsy and Parkinson's disease. Neuroimage 2004;23: 663-69 CrossRef Medline

6. Erbetta A, Mandelli ML, Savoiardo M, et al. Diffusion tensor imaging shows different topographic involvement of the thalamus in progressive supranuclear palsy and corticobasal degeneration. AJNR Am J Neuroradiol 2009;30:1482-87 CrossRef Medline

7. Nilsson C, Markenroth Bloch K, Brockstedt S, et al. Tracking the neurodegeneration of parkinsonian disorders: a pilot study. $\mathrm{Neu}$ roradiology 2007;49:111-19 CrossRef Medline

8. Seppi K, Poewe W. Brain magnetic resonance imaging techniques in the diagnosis of parkinsonian syndromes. Neuroimage Clin N Am 2010;20:29-55 CrossRef Medline

9. Canu E, Agosta F, Baglio F, et al. Diffusion tensor magnetic resonance imaging tractography in progressive supranuclear palsy. Mov Disord 2011;26:1752-55 CrossRef Medline

10. Knake S, Belke M, Menzler K, et al. In vivo demonstration of microstructural brain pathology in progressive supranuclear palsy: a DTI study using TBSS. Mov Disord 2010;25:1232-38 CrossRef Medline

11. Padovani A, Borroni B, Brambati SM, et al. Diffusion tensor imaging and voxel based morphometry study in early progressive supranuclear palsy. J Neurol Neurosurg Psychiatry 2006;77:457-63 CrossRef Medline

12. Wang J, Wai Y, Lin WY, et al. Microstructural changes in patients with progressive supranuclear palsy: a diffusion tensor imaging study. J Magn Reson Imaging 2010;32:69-75 CrossRef Medline

13. Whitwell JL, Master AV, Avula R, et al. Clinical correlates of white matter tract degeneration in progressive supranuclear palsy. Arch Neurol 2011;68:753-60 CrossRef Medline

14. Longoni G, Agosta F, Kostić VS, et al. MRI measurements of brainstem structures in patients with Richardson's syndrome. Progressive supranuclear palsy-parkinsonism, and Parkinson's disease. Mov Disord 2011;26:247-55 CrossRef Medline

15. Agosta F, Pievani M, Svetel M, et al. Diffusion tensor MRI contributes to differentiate Richardson's syndrome from PSP-parkinsonism. Neurobiol Aging 2012;33:2817-26 CrossRef Medline

16. Jankovic J, Rajput AH, McDermott MP, et al. The evolution of diagnosis in early Parkinson disease: Parkinson Study Group. Arch Neurol 2000;57:369-72 CrossRef Medline

17. Litvan I, Agid Y, Calne D, et al. Clinical research criteria for the diagnosis of progressive supranuclear palsy (Steele-RichardsonOlszewski syndrome): report of the NINDS-SPSP international workshop. Neurology 1996;47:1-9 CrossRef Medline

18. Calne DB, Snow BJ, Lee C. Criteria for diagnosing Parkinson's disease. Ann Neurol 1992;32(suppl):S125-27 CrossRef Medline

19. Fahn S, Elton R, members of the UPDRS Development Committee. Unified Parkinson's Disease Rating Scale. In: Fahn S, Marsden CD, Calne DB, Goldstein M, eds. Recent Developments in Parkinson's Disease. Florham Park, NJ: Macmillan Healthcare Information; 1987:153-64

20. Hoehn MM, Yahr MD. Parkinsonism: onset, progression and mortality. Neurology 1967;17:427-42 CrossRef Medline

21. Folstein MF, Folstein SE, McHugh PR. 'Mini-Mental State': a practical method for grading the cognitive state of patients for the clinician. J Psychiatry Res 1975;12:189-98 CrossRef Medline

22. Jenkinson M, Beckmann CF, Behrens TE, et al. FSL. Neuroimage 2012;62:782-90 CrossRef Medline

23. Smith SM, De Stefano N, Jenkinson M, et al. Normalized accurate measurement of longitudinal brain change. J Comput Assist Tomogr 2001;25:466-75 CrossRef Medline

24. Smith SM, Zhang Y, Jenkinson M, et al. Accurate, robust and automated longitudinal and cross-sectional brain change analysis. $\mathrm{Neu}$ roimage 2002;17:479-89 CrossRef Medline

25. Smith SM. Fast robust automated brain extraction. Hum Brain Mapp 2002;17:143-55 CrossRef Medline

26. Cherubini A, Péran P, Caltagirone C, et al. Aging of subcortical nuclei: microstructural, mineralization and atrophy modifications 
measured in vivo using MRI. Neuroimage 2009;48:29-36 CrossRef Medline

27. Thiebaut de Schotten M, Ffytche DH, Bizzi, et al. Atlasing location, asymmetry and inter-subject variability of white matter tracts in the human brain with MR diffusion tractography. Neuroimage 2011;54 1:49-59 CrossRef Medline

28. Ohtsuka K, Igarashi Y, Chiba S. Cerebellar peduncle lesion without saccadic abnormalities. Ophthalmologica 1992;204:44-48 CrossRef Medline

29. Nicoletti G, Tonon C, Lodi R, et al. Apparent diffusion coefficient of the superior cerebellar peduncle differentiates progressive supranuclear palsy from Parkinson's disease. Mov Disord 2008;23: 2370-6 CrossRef Medline

30. Nicoletti G, Rizzo G, Barbagallo G, et al. Diffusivity of cerebellar hemispheres enables discrimination of cerebellar or parkinsonian multiple system atrophy from progressive supranuclear palsyRichardson syndrome and Parkinson disease. Radiology 2013;267: 843-50. CrossRef Medline

31. Quattrone A, Nicoletti G, Messina D, et al. MR imaging index for differentiation of progressive supranuclear palsy from Parkinson disease and the Parkinson variant of multiple system atrophy. $R a$ diology 2008;246:214-21 CrossRef Medline

32. William DR, de Silva R, Paviour DC, et al. Characteristics of two distinct clinical phenotypes in pathologically proven progressive supranuclear palsy: Richardson's syndrome and PSP-parkinsonism. Brain 2005;128:1247-58 CrossRef Medline

33. Agosta F, Kostic VS, Galantucci S, et al. The in vivo distribution of brain tissue loss in Richardson's syndrome and PSP-parkinsonism: a VBM-DARTEL study. Eur J Neurosci 2010;32:640-47 CrossRef Medline

34. Worker A, Blain C, Jarosz J, et al. Diffusion tensor imaging of Parkinson disease, multiple system atrophy and progressive supranuclear palsy: a tract-based spatial statistics study. Plos One 2014;9: e112638 CrossRef Medline

35. Meijer FJ, van Rumund A, Tuladhar A, et al. Conventional 3T brain MRI and diffusion tensor imaging in the diagnostic workup of early stage parkinsonism. Neuroradiology 2015;57:655-69 CrossRef Medline

36. Douaud G, Behrens TE, Poupon C, et al. In vivo evidence for the selective subcortical degeneration in Huntington's disease. Neuroimage 2009;46:958-66 Medline 